\title{
Comportement de 7 variétés de haricot volubile dans trois territoires de la province du Sud Kivu en R. D. Congo et recherche de la meilleure saison de culture
}

\author{
Walangululu Masamba Jean, Muhindo Iragi Daniel, Baluku Safari Patrick, Cito Miderho \\ Christus, Lyadunga Mulumeoderhwa Janvier, Sebakara, Shandwa Emile et Mvula, Hugues \\ Université Catholique de Bukavu, B.P. 2, Cyangugu, Rwanda \\ Adresse de courrier électronique:W alangululu Masamba,walangululu@yahoo.fr \\ Mots clés: haricot volubile, rendement, Kavumu, Mulamba, Kalehe \\ Key words: climbing beans, yield, Kalehe, Kavumu, Mulamba \\ Publication date 30/09/2019, http://www.m.elewa.org/JAPS
}

\section{RESUME}

Les variétés de haricot volubile Namulenga et CODMLV 059 avaient été introduites dans les essais effectués dans les groupements Mushinga du territoire de Walungu et Bushumba du territoire de Kabare dans le cadre du projet Legume choice, dans l'intention de rechercher de nouvelles niches pour le haricot volubile; les résultats ont montré que les deux variétés se sont très bien comportées et ont montré des rendements appréciés de la part des paysans de ces groupements, un peu plus pour CODMLV 059 dans le territoire de Kabare et un peu plus pour Namulenga en territoire de Walungu. L'inclusion de la variété Namulenga dans les travaux de dissémination du paquet technologique conçu par la faculté d'agronomie de l'Université Catholique de Bukavu (U.C.B.) dans le groupement Mulamba en territoire de Walungu, a donné un rendement inférieur par rapport au potentiel de cette variété. Il a alors été pensé entre plusieurs suppositions que cette variété n'était certainement pas adaptée dans ce groupement. Le paquet technologique est basé sur une association manioc-légumineuses ou manioc-maïs ou maïs- légumineuses selon les saisons et les sites, en y incorporant les engrais minéraux et organiques. Ainsi en marge des essais de dissémination du paquet technologique du projet VLIR à Mulamba en territoires de Walungu, à Kavumu, en territoire de Kabare et de Kalehe en territoire de Kalehe, il a été entrepris ce travail de comportement de 7 variétés de haricot volubile dans ces milieux afin de choisir une variété à incorporer dans les essais de dissémination en vue d'augmenter la production de haricot dans le Sud Kivu et identifier la meilleure saison de culture. Ce travail a été conduit en deux saisons successives en 2017 (octobre à décembre) et 2018 (janvier à juillet) sur un dispositif en blocs randomisés avec trois répétitions en utilisant les variétés Namulenga, Nyiramuhondo, CODMLV 059, Nain de Kyondo, Cuarentino 0817, M211 et Puebla Criolla. Les paramètres végétatifs et de rendement ont été observés. Les résultats ont montré que les paramètres végétatifs (hauteur des plants et nombre de feuilles) et ceux de rendement étaient plus élevés en saison B qu'en saison A et que dès lors, la saison B peut être considérée comme la plus favorable. Sur le plan du rendement, il y a eu des variations allant de 0,878 à 4,960 t/ha en saison $B$, la meilleure saison, selon les sites mais les variétés suivantes peuvent être recommandées: Nyiramuhondo, Namulenga et Puebla Criolla, pourvu que bien entendu elles puissent présenter de bonnes qualités culinaires et sensorielles. 


\section{ABSTRACT}

In the framework of legume choice project, Namulenga and CODMLV 059 climbing bean varieties were introduced in Mushinga administrative area, Walungu territory, and in Bushumba administrative area, Kabare territory, as niche exploration for climbing beans. Results showed that performance of both varieties was not very different although a trend of CODMLV 059 performing better in the Bushumba site and Namulenga giving slightly better performance in Mushinga; these varieties were appreciated and adopted by farmers of these areas. When introduced in the dissemination of project package of VLIR project of the faculty of agriculture, Catholic University of Bukavu, in Mulamba administrative area, the yield of Namulenga climbing bean was low compared to its potential. It was thought among many thought that this variety is not adapted in that area. The project package is scaling up an association of cassava and legumes or cassava- legumes and maize or maize and cassava with farm yard manure and fertilizer in Mulamba, Kalehe and Kavumu administrative areas, in order to increase legume production. Therefore, it was decided that beside dissemination trials to carry out other trials in all three sites, comparing seven climbing beans varieties in order to select best one to be included in the dissemination trials. This work was carried out in two consecutive cropping seasons: B season (February to July) in 2018 and A season (October to December) in 2017, on a randomized bloc design with three replications, using Namulenga, Nyiramuhondo, CODMLV 059, Nain de Kyondo, Cuarentino 0817, M211 and Puebla Criolla varieties, in order to also find the best cropping season. Observations were made on vegetative parameters (percent germination, plant height, collar diameter and leaf number) and production parameters (weight of hundred grains, number of pods per plant, number of grain per pod and yield). Results revealed that vegetative parameters (plant height and leaf number) and those of yield were higher in B season (January to July 2018) than in A season (October to December 2017), suggesting therefore that B season is the best cropping season for climbing beans. Yield varied between 0.878 and 4.960 tons per hectare in the best season, according to sites but following varieties can be recommended, provide their cooking and taste qualities: Nyiramuhondo, Namulenga and Puebla Criolla.

\section{INTRODUCTION}

Le haricot représenterait à lui seul près de $33 \%$ de la production mondiale de légumineuses, ce qui fait de lui la légumineuse la plus consommée dans le monde (Vogel, 2017). En République Démocratique du Congo (RDC) c'est dans les provinces du Nord et du Sud Kivu qu'il est le plus produit et consommé, sa consommation est estimée à $300 \mathrm{~g}$ par habitant par jour (Harvestplus, 2009 ; Civava, 2013). En plus des graines consommées de diverses manières, les feuilles de haricot sont consommées comme légume au Sud Kivu. Les besoins de la province du Sud Kivu sont estimés à 155.676 tonnes, contre une production de 65.338 tonnes, le déficit dû à diverses causes étant comblé par des importations du Nord Kivu et du Rwanda voisin (Vwima, 2014). Outre son importance sur le plan alimentaire, grâce à sa richesse en protéines, en fibres et en vitamines, qui font de lui « la viande du pauvre » (Robertson, 2017), le haricot a une importance agronomique par sa capacité, en tant que légumineuse, de fixer l'azote atmosphérique, de l'utiliser pour sa propre croissance, d'enrichir ainsi le sol en azote et de diminuer le recours à la fertilisation azotée, ce qui procure de meilleures récoltes pour les plantes cultivées après sa récolte sur le même terrain (Nieuwenhuis et Nieuwelink, 2005). On distingue, selon Baudouin et al (2001), deux habitus de croissance chez le haricot, avec pour chaque habitus plusieurs formes suivant le type de développement de la partie terminale de la tige principale, suivant le 
nombre de nœuds, suivant la longueur des entre-nœuds et, en conséquence, la hauteur de la plante, suivant l'aptitude à grimper et suivant le degré et le type de ramification: habitus de croissance apparemment déterminée avec deux types, tous de forme naine et habitus de croissance indéterminée, avec aussi deux types de plantes, toutes volubiles. Sur le plan du rendement, les mêmes auteurs renseignent des écarts énormes entre les systèmes traditionnels et modernes et entre les haricots nains et les volubiles: 200 à 500, voire $3000 \mathrm{~kg} / \mathrm{ha}$ de graines sèches avec des cultivars améliorés pour les haricots nains et $6000 \mathrm{~kg}$ pour les haricots volubiles. Le remplacement progressif du haricot nain par le haricot volubile est une des solutions envisageables pour augmenter la production de haricot au Sud Kivu, ce qui a été introduit dans le projet legume choice à Mushinga et Bushumba. Au Sud Kivu, le haricot est rarement produit en pur, il est souvent associé au maïs, manioc et autres cultures. Les variétés de haricot volubile Namulenga et CODMLV 059 ont été introduites dans les essais effectués dans les groupements Mushinga du territoire de Walungu et Bushumba, en territoire de Kabare, dans le cadre du projet Legume choice pour booster la production de haricot dans ce groupement; ces variétés s'y sont très bien comportées et ont montré des rendements appréciés de la part des paysans (Walangululu et

\section{MATERIEL ET METHODES}

3.1 Milieu. Ce travail a été réalisé en saisons B (janvier à juillet) 2018 et A (août à décembre) en 2017 à Mulamba, dans le groupement de Mulamba, territoire de Walungu, à Kavumu dans le groupement Bughore en territoire de Kabare et à Ihusi et Murambi dans le groupement Mbinga Sud dans le territoire de Kalehe. Les sites d'essais sont al,. 2017) L'inclusion de la variété Namulenmga dans les travaux de dissémination du paquet technologique conçu par la faculté d'agronomie de l'Université Catholique de Bukavu (U.C.B.) dans le groupement Mulamba en territoire de Walungu, dans le cadre du projet VLIR n'a pas montré des résultats escomptés dans ce groupement. Il a alors été pensé entre autres que cette variété n'était certainement pas adaptée dans ce groupement. Le paquet technologique est basé sur une association manioc-légumineuses ou manioc-maïs ou maïslégumineuses selon les saisons et les sites, en y incorporant les engrais minéraux et organiques. Ainsi en marge des essais de dissémination du paquet technologique du projet VLIR à Mulamba en territoire de Walungu, à Kavumu, en territoire de Kabare et à Kalehe, en territoire de Kalehe, ce travail a été entrepris en 2017 et 2018 dans le but de comparer 7 variétés de haricot volubile au cours de deux saisons culturales successives en vue d'identifier celle ou celles qui s'adapteraient mieux dans ces groupements et donneraient un rendement élevé en vue de les inclure dans le paquet technologique pour réduire les écarts entre la production de haricot dans la province et les besoins. Ce travail, effectué en deux saisons successives avait aussi comme autre objectif de déterminer la meilleure saison de culture du haricot volubile dans ces groupements.

situés à des altitudes respectives de $1762 \mathrm{~m}$ pour Kavumu, $1473 \mathrm{~m}$ pour Mulamba et 1583 $\mathrm{m}$ pour Kalehe. L'altitude élevée des sites détermine le climat frais qu'affectionne le haricot. Les données pluviométriques des sites d'essai au cours des deux saisons culturales sont fournies au tableau 1 et quelques caractéristiques du sol des sites au tableau 2 . 
Tableau 1 : Données pluviométriques pendant les essais

\begin{tabular}{|l|l|l|l|l|l|l|}
\hline \multirow{2}{*}{ Mois } & Kavumu & Mulamba & \multicolumn{2}{l|}{ Kalehe } \\
\cline { 2 - 7 } & Total $\mathbf{( m m})$ & N J P & Total $\mathbf{( m m})$ & N J P & Total $(\mathbf{m m})$ & N J P \\
\hline Octobre 2017 & 180,4 & 20 & 93,0 & 13 & 173 & 19 \\
\hline Novembre & 178,7 & 22 & 179,0 & 17 & 176 & 24 \\
\hline Décembre & 129,7 & 12 & 155,0 & 17 & 168 & 13 \\
\hline Janvier 2018 & 19,4 & 17 & 123,0 & 20 & 146 & 11 \\
\hline Février & 203,7 & 19 & 122,0 & 15 & 98 & 8 \\
\hline Mars & 107,9 & 19 & 89,0 & 14 & 161 & 20 \\
\hline Avril & 264,9 & 16 & 191,0 & 24 & 215 & 22 \\
\hline Mai & 170,5 & 16 & 67,3 & 11 & 182 & 12 \\
\hline Juin & 14,9 & 3 & 0 & 0 & 58 & 8 \\
\hline
\end{tabular}

NJP : nombre de jours de pluies

Il résulte de ces données que les données pluviométriques étaient normales et que dans l'ensemble des deux saisons et dans les trois sites la quantité totale des pluies a répondu aux exigences du haricot, qui sont de 300 à $400 \mathrm{~mm}$ d'eau, bien distribuées, modérées, car le haricot craint l'excès de pluies et d'humidité atmosphérique (Baudouin et al, 2001, Carburet et Hekimian, 2003); la distribution a été presque la même pour chaque site en comparant les saisons. Cependant, il y a eu plus de pluies pendant la période des semis en en saison B (février-mars) qu'en saison $A$, ce qui peut nuire au bon développement du haricot et plus de pluies pendant la floraison et la fructification en saison B qu'en saison $A$, ce qui peut aussi nuire au rendement du haricot.

Tableau 2. Quelques caractéristiques chimiques du sol des sites d'essais

\begin{tabular}{|l|l|l|l|}
\hline & Kavumu & Mulamba & Kalehe \\
\hline $\mathrm{pH}$ & 5,43 & 4,46 & 5,48 \\
\hline $\mathrm{C}(\%)$ & 3,089 & 3,48 & 2,81 \\
\hline $\mathrm{N}(\%)$ & 0,34 & 0,27 & 0,28 \\
\hline $\mathrm{P}(\mathrm{mg} / \mathrm{kg})$ & 7,6 & 24,44 & 16,75 \\
\hline
\end{tabular}

En ce qui concerne le $\mathrm{pH}$, celui-ci est au seuil critique inférieur du haricot $(5,0-8,1)$ à Kavumu et Kalehe mais non convenable au haricot à Mulamba, où le sol est acide. Pour ce qui est de la teneur en carbone, elle est acceptable dans les trois sites, car supérieure au seuil de 0,8\% (Baudouin et al., 2011). La teneur en azote est faible à Mulamba et Kalehe, car inférieure au seuil de $0,3 \%$; cependant, dans un sol déficient, le haricot, par sa capacité de fixer l'azote atmosphérique à travers les nodosités des racines, enrichit le sol en azote. En dehors de Kavumu où la teneur en phosphore total est déficiente, faible, elle est acceptable dans les deux autres sites car supérieure à $15 \mathrm{mg} / \mathrm{kg}$; le phosphore joue un grand rôle dans la croissance racinaire du haricot et dans la fixation biologique de l'azote chez le haricot. La plupart des sols tropicaux étant pauvres en phosphore assimilable, du phosphore a été ajouté aux essais.

3.2 Matériel. Le matériel végétal utilisé dans ce travail comprenait 7 variétés de haricot volubile reçues de Harvestplus; il s'agit de: M 211, NAMULENGFA, CUARENTINO 0817, PUEBLA, CRIOLLA, CODMLV 059, NYIRAMUHONDO et NAIN DE KYONDO. Les caractéristiques de ces variétés sont reprises au tableau 3. 
Tableau 3 : Caractéristiques des variétés en étude

\begin{tabular}{|c|c|c|c|c|c|c|c|}
\hline \multirow[t]{2}{*}{ Variétés } & \multirow{2}{*}{$\begin{array}{l}\text { Jours } \\
\text { maturité } \\
\text { physi. }\end{array}$} & \multirow{2}{*}{$\begin{array}{l}\text { Poids } \\
100 \\
\text { graines } \\
\text { (g) }\end{array}$} & \multicolumn{2}{|c|}{$\begin{array}{l}\text { Rendement } \\
\text { (t/ha) }\end{array}$} & \multicolumn{3}{|c|}{ Résistance aux maladies } \\
\hline & & & $\begin{array}{l}\text { En } \\
\text { station }\end{array}$ & $\begin{array}{l}\text { En } \\
\text { milieu } \\
\text { réel }\end{array}$ & $\begin{array}{l}\text { Taches } \\
\text { angul. }\end{array}$ & Ascochytose & Anthracnose \\
\hline CODMLV 059 & $95-100$ & $48-52$ & $2,5-3,5$ & $1,0-1,6$ & Resist. & Resist. & Resist. \\
\hline $\begin{array}{l}\text { Cuarentino } \\
0817\end{array}$ & $95-100$ & $24-28$ & $2,5-3,5$ & $1,0-1,6$ & Resist. & Resist. & Resist. \\
\hline Namulenga & $75-80$ & $30-32$ & $2,5-4,0$ & $1,2-2,0$ & Resist. & Resist. & Resist. \\
\hline Puebla Criollo & $100-105$ & $38-42$ & $2,5-3.0$ & $1,4-1,8$ & Resist. & Resist. & Resist. \\
\hline M 211 & $95-100$ & $23-25$ & $2,0-3,0$ & $1,0-1,8$ & Resist. & Resist. & Resist. \\
\hline $\begin{array}{l}\text { Nain de } \\
\text { Kyondo }\end{array}$ & $75-80$ & $20-25$ & $2,0-2,5$ & $1,0-1,5$ & Resist. & Interm, & Resist. \\
\hline Nyiramuhondo & $105-110$ & $35-38$ & $3,0-4,0$ & $1,5-2,0$ & Resist. & Resist. & Resist. \\
\hline
\end{tabular}

Source : Institut National pour l'Etude et la Recherche Agronomique (INERA), 2006.

Il résulte de ces caractéristiques que les variétés Namulenga et Nain de Kyondo sont plus précoces que toutes les autres et que la variété Nyiramuhondo est la plus tardive; en milieu réel, la variété Nyiramuhondo est la plus productive, suivie de Puebla Criolla et Namulenga. Toutes les variétés résistent aux maladies les plus courantes, c'est-à-dire les taches anguleuses, l'ascochytose et l'anthracnose, à part la variété Nain de Kyondo qui a une résistance intermédiaire à l'ascochytose.

3.3 Méthode. Ces 7 variétés ont été mises en comparaison dans un dispositif en blocs aléatoires complets en trois répétitions en saisons B en 2017 et A en 2018. Compte tenu de la composition des sols, des fertilisants ont été appliqués au second labour, utilisant le DAP comme source de phosphore et la matière organique, dans les proportions de $100 \mathrm{~kg}$ de $\mathrm{P}$ et 1 tonne de matière organique par hectare. Les semis ont eu lieu le 17 octobre 2017 et le 10 mars 2018 pour Kavumu, le 13 novembre 2017 et le15 mars 2018 pour Mulamba et le 22 octobre et le 28 février pour Kalehe. Les vides ont été regarnis après le semis et la lutte contre les adventices a été faite par des sarclages. Deux semaines après le semis les plants ont reçu des tuteurs de $2 \mathrm{~m}$. Les paramètres végétatifs et de rendement ont été prélevés et mesurés; il s'agit du taux de germination, de la hauteur des plants et de leur diamètre au collet, du nombre de feuilles et de leur surface foliaire, pour les paramètres végétatifs et du nombre de gousses par plant, le nombre de graines par gousse, le poids de 100 graines et le rendement, pour les paramètres du rendement. Dans les résultats, il n'a été tenu compte que des paramètres végétatifs et de rendement pouvant beaucoup influencer la croissance et le rendement. L'analyse de la variance à un et deux critères de classification a été utilisée et la séparation des moyennes par la plus petite différence significative. Les variétés CODMLV 059 et Nain de Kyondo ont été comparées aux autres au cours de la saison où elles étaient en essai par le test t de Student. 


\section{4}

\subsection{Paramètres végétatifs}

4.1.1 Taux de levée des plants par variété, par saison et par site. Le taux de levée des plants par variété, par saison et par site est fourni au tableau 4.

Tableau 4 : Taux de levée des variétés en étude (en \%)

\begin{tabular}{|l|l|l|l|l|l|l|}
\hline \multirow{2}{*}{ Variétés } & \multicolumn{2}{l|}{ Mulamba } & \multicolumn{2}{l|}{ Kavumu } & \multicolumn{2}{l|}{ Kalehe } \\
\cline { 2 - 7 } & Saison A & Saison B & Saison A & Saison B & Saison A & Saison B \\
\hline M 211 & 64,12 & 61,25 & 65,70 & 98,13 & 84,58 & 70,00 \\
\hline Cuarantino 0817 & 82,13 & 53,75 & 69,38 & 96,73 & 81,67 & 76,19 \\
\hline Namulenga & 78,75 & 50,88 & 73,06 & 96,60 & 91,25 & 75,23 \\
\hline Puebla Criolla & 71,63 & 60,00 & 81,63 & 96.23 & 83,75 & 60,00 \\
\hline Nyiramuhondo & 86,63 & 64,12 & 65,89 & 94,76 & 87,08 & 52,38 \\
\hline CODMLV 059 & & 53,75 & & 96,56 & & 62,85 \\
\hline Nain de Kyondo & 94,12 & & 69,00 & & 77,50 & \\
\hline
\end{tabular}

En dehors du groupement de Ihusi à Kalehe en territoire de Kalehe et de Mulamba dans le territoire de Walungu où le taux de germination des graines était supérieur en saison A par rapport à la saison $\mathrm{B}$, le taux de germination des graines en saison A 2017 a été inferieur dans le groupement Bugorhe à Kavumu, en territoire de Kabare, par rapport à la saison B 2018 ; ceci pourrait être expliqué par les conditions de conservation des semences, la pluviométrie ayant été normale au cours des deux saisons. La variété CODMLV 059 n'ayant pas bien germé en saison A malgré le regarnissage des vides, elle a été exclue de l'expérimentation au cours de cette saison; la variété Nain de Kyondo n'a pas été disponible chez Harvestplus en saison $\mathrm{B}$, les graines issues de la saison $\mathrm{A}$ ayant manifesté un grand retard dans la germination. 4.1.2 Hauteur moyenne des plants par variété, par saison et par site. La hauteur moyenne des plants par variété, par saison et par site est fournie dans le tableau 5.

Tableau 5 : Hauteur moyenne des plants (en $\mathrm{cm}$ )

\begin{tabular}{|l|l|l|l|l|l|l|}
\hline \multirow{2}{*}{ Variétés } & \multicolumn{2}{l|}{ Mulamba } & \multicolumn{2}{l|}{ Kavumu } & \multicolumn{2}{l|}{ Kalehe } \\
\cline { 2 - 7 } & Saison A & Saison B & Saison A & Saison B & Saison A & Saison B \\
\hline M 211 & $95,37 \mathrm{~b}$ & 181,83 & 164,8 & 234,0 & 195,4 & 234,1 \\
\hline Cuarantino 0817 & $96,40 \mathrm{~b}$ & 156,37 & 149,5 & 260,3 & 194,8 & 237,3 \\
\hline Namulenga & $174,50 \mathrm{a}$ & 199,90 & 239,8 & $279,8 \mathrm{a}$ & 203,7 & 244,9 \\
\hline Puebla Criolla & $162,77 \mathrm{a}$ & 199,73 & 212,3 & $262,0 \mathrm{a}$ & 197,1 & 264,8 \\
\hline Nyiramuhondo & $145,10 \mathrm{a}$ & 203,53 & 212,3 & $306,2 \mathrm{a}$ & 222,6 & 243,1 \\
\hline CODMLV 059 & & 197,93 & & 304,9 & & 245,4 \\
\hline Nain de Kyondo & 113,97 & & 153,6 & & 181,2 & \\
\hline
\end{tabular}

La hauteur moyenne des plants a été supérieure en saison B 2018 par rapport à la saison A 2017 sur tous les sites. Ceci peut être explique par les conditions environnementales (pluies et meilleure répartition) plus favorables au cours de cette saison; cette saison serait donc la meilleure du point de vue végétation du haricot volubile dans ces sites. L'analyse de la variance de ces données a, pour Mulamba, révélé des différences significatives de hauteur des plants pour la saison A, tandis qu'il n'y a pas eu de différence en saison B ; en saison A, les plants des variétés Namulenga, Puebla Criolla et Nyiramuhondo ont été plus hauts que ceux des 
autres variétés. Pour ce qui est de Kavumu, la comparaison des moyennes après l'analyse de la variance a donné la même configuration qu'a Mulamba. Pour ce qui est de Kalehe, seule une différence entre saison a été enregistrée. La supériorité de la hauteur des plants en saison B peut être expliquée par plus de pluies au cours de cette saison, ce qui augmente la croissance ; le haricot volubile ayant une croissance indéterminée, plus le plant sera haut, plus il y aura des feuilles et des fleurs et plus élevé pourra être le rendement par voie de conséquence. Les différences entre variétés ne peuvent être expliquées que par le génotype propre de chaque variété.

4.1.3 Nombre de feuilles. Le nombre de feuilles par plant 10 à 11 semaines après semis des variétés en étude par saison et par site est donne au tableau 6.

Tableau 6 : Nombre de feuilles par plant

\begin{tabular}{|l|l|l|l|l|l|l|}
\hline \multirow{2}{*}{ Variétés } & Mulamba & \multicolumn{2}{l|}{ Kavumu } & \multicolumn{2}{l|}{ Kalehe } \\
\cline { 2 - 7 } & Saison A & Saison B & Saison A & Saison B & Saison A & Saison B \\
\hline M 211 & 9,91 & 21,08 & 24,5 & 16,5 & 33,3 & 38,5 \\
\hline Cuarantino 0817 & 10,47 & 25,49 & 18,6 & 15,2 & 49,1 & 42,1 \\
\hline Namulenga & 11,86 & 19,64 & 16,7 & 17,6 & 20,4 & 35,3 \\
\hline Puebla Criolla & 14,71 & 25,04 & 21,9 & 18,8 & 43,3 & 46,2 \\
\hline Nyiramuhondo & 12,50 & 25,69 & 24,4 & 15,7 & 39,7 & 37,5 \\
\hline CODMLV 059 & & 20,87 & & 15,5 & & 34,8 \\
\hline Nain de Kyondo & 10,28 & & 21,7 & & 36,4 & \\
\hline
\end{tabular}

Le nombre de feuilles dans les sites de Mulamba et Kalehe a été plus élevé en saison B qu'en saison $A$, ce qui est contraire pour Kavumu; la situation de Kavumu peut être expliquée par trop de pluies au troisième mois de la culture. La séparation des moyennes au niveau de l"interaction après l'analyse de la variance a révélé pour Kavumu que les variétés ont le même nombre de feuilles en saison A. A Mulamba la séparation des moyennes après l'analyse de la variance a montré que les plants des variétés Puebla Criolla, Namulenga et Nyiramuhondo ont porté plus de feuilles que ceux des autres plants en saison A, tandis qu'en saison B, il n'y avait aucune différence entre les variétés. A Kalehe la séparation des moyennes au niveau de l'interaction variété-saison après analyse de la variance a montré que 3 variétés
(Cuarantino 0817, Puebla Criolla, Nyiramuhondo) en saison A avaient le même nombre de feuilles que 5 variétés en saison $\mathrm{B}$ (Cuarantino 0817, Puebla Criolla, Nyiramuhondo, Namulenga et M 211). Le feuillage a un rôle déterminant dans la phase végétative et reproductive car constituant les principaux moteurs de la croissance suite à l'activité photosynthétique et entraine une corrélation positive sur le rendement; cependant un très grand nombre de feuilles baisse le rendement car la respiration gaspille une part d'assimilas photosynthétiques (Du Jardin, 1994).

\subsection{Paramètres de rendement}

4.2.1 Poids de $\mathbf{1 0 0}$ graines. Le poids de 100 graines à la récolte des variétés par saison et par site est présenté au tableau 7. 
Tableau 7 : Poids de 100 graines (en grammes)

\begin{tabular}{|l|l|l|l|l|l|l|}
\hline \multirow{2}{*}{ Variétés } & \multicolumn{2}{|l|}{ Mulamba } & \multicolumn{2}{l|}{ Kavumu } & \multicolumn{2}{l|}{ Kalehe } \\
\cline { 2 - 7 } & Saison A & Saison B & Saison A & Saison B & Saison A & Saison B \\
\hline M 211 & 24,47 & 28,40 & 31,3 & 35,4 & 30,63 & 38,24 \\
\hline Cuarantino 0817 & 28,53 & 29,38 & 26,4 & 35,5 & 27,34 & 29,34 \\
\hline Namulenga & 31,66 & 34,53 & 38,8 & 43,0 & 26,54 & 52,40 \\
\hline Puebla Criolla & 44,60 & 41,59 & 41,2 & 44,8 & 41,88 & 32,74 \\
\hline Nyiramuhondo & 36,85 & 36,52 & 33,6 & 39,4 & 33,81 & 43,16 \\
\hline CODMLV 059 & & 42,02 & & 62,7 & & 51,33 \\
\hline Nain de Kyondo & 28,65 & & 24,9 & & 30,23 & \\
\hline
\end{tabular}

Il ressort du poids de 100 graines des variétés en étude que celui-ci a été un peu plus élevé en saison B qu'en saison A, influencé certainement par les conditions de croissance meilleures en saison $\mathrm{B}$, même s'il est admis que ce paramètre est variétal. A Mulamba, la comparaison des moyennes de ce paramètre après analyse de la variance a révélé qu'en saison A le poids de 100 graines de la variété Puebla Criolla est plus élevé que celui de la variété Nyiramuhondo qui la suit, tandis qu'en saison B c'est celui des variétés CODMLV 059 et Puebla Criolla qui est suivi par les variétés Nyiramuhondo et Namulenga. A l'exception de la variété CODMLV 059 qui a enregistré un poids de 100 graines inferieur au potentiel (tableau 1), tous les autres sont dans la marge ou sont un peu supérieurs. A Kavumu, les variétés Puebla Criolla et Namulenga ont un poids de 100 graines plus élevé que la variété Nyiramuhondo qui les suit. Comparativement au potentiel, la même constatation qu'a Mulamba est faite ici, à l'exception de la variété CODMLV 059 qui a un poids plus élevé. A Kalehe, la séparation des moyennes au niveau de l'interaction après analyse de la variance a montré que la variété Puebla Criolla avait un poids de 100 graines supérieur à celui de Namulenga en saison B, Puebla Criolla en saison A et Nyiramuhondo en saison B. Comparativement au potentiel, le constat de Kavumu est le même à Kalehe. Les différences au sein des variétés sont dues à la variabilité génétique des variétés mais les conditions climatiques peuvent l'influencer (Tsibingu et al., 2017).

4.2.2 Nombre de gousses/plant. Le nombre de gousses par plant des variétés par saison et par site est présenté au tableau 8.

Tableau 8 : Nombre de gousses/plant

\begin{tabular}{|l|l|l|l|l|l|l|}
\hline \multirow{2}{*}{ Variétés } & \multicolumn{3}{|l|}{ Mulamba } & \multicolumn{2}{l|}{ Kavumu } & \multicolumn{2}{l|}{ Kalehe } \\
\cline { 2 - 7 } & Saison A & Saison B & Saison A & Saison B & Saison A & Saison B \\
\hline M 211 & 4,33 & 24,33 & 16,58 & 20,88 & 13,52 & 19,29 \\
\hline Cuarantino 0817 & 3,43 & 26,53 & 16,16 & 21,3 & 10,92 & 21,98 \\
\hline Namulenga & 5,83 & 18,47 & 22,06 & 24,16 & 12,82 & 19,45 \\
\hline Puebla Criolla & 5,80 & 24,67 & 10,53 & 11,4 & 11,01 & 23,27 \\
\hline Nyiramuhondo & 3,43 & 35,40 & 16,3 & 22,96 & 8,98 & 23,90 \\
\hline CODMLV 059 & & 19,07 & & 0,76 & & 18,90 \\
\hline Nain de Kyondo & 5,00 & & 34,14 & & 16,57 & \\
\hline
\end{tabular}

Le nombre de gousses par plant a été plus élevé en saison B qu'en saison A, cela dans tous les sites, suite aux conditions de croissance meilleures au cours de cette saison. Le nombre de gousses par plant est un paramètre qui est directement lié au rendement en graines; quoiqu'étant une caractéristique variétale, il peut être affecté par les facteurs de 
l'environnement. Ce nombre n'a pas varié en saison A à Mulamba, tandis qu'il a varié entre les variétés en saison $\mathrm{B}$ et la comparaison des moyennes a montré que la variété Nyiramuhondo a enregistré plus de gousses que les autres variétés qui s'équivalent. A Kavumu, l'interaction variété-saison après analyse de la variance a montré plus de gousses pour les variétés Namulenga, Cuarantino 0817 et M 211 en saison B et Namulenga en saison A par rapport aux autres variétés en saisons A et B. La séparation des moyennes au niveau de l'interaction variété-saison à Kalehe après analyse de la variance, a montré un plus grand nombre de gousses par plant pour toutes les variétés en saison $B$.

4.2.3 Nombre de graines/gousse. Le nombre de graines par gousse par variété, par saison et par site est fourni au tableau 9.

Tableau 9: Nombre de graines/gousse des variétés par saison et par site

\begin{tabular}{|l|l|l|l|l|l|l|}
\hline \multirow{2}{*}{ Variétés } & \multicolumn{2}{|l|}{ Mulamba } & \multicolumn{2}{l|}{ Kavumu } & \multicolumn{2}{l|}{ Kalehe } \\
\cline { 2 - 7 } & Saison A & Saison B & Saison A & Saison B & Saison A & Saison B \\
\hline M 211 & 3,77 & 6,20 & 5,57 & 9,88 & 4,56 & 8,46 \\
\hline Cuarantino 0817 & 4,33 & 5,87 & 6,23 & 8,41 & 5,26 & 8,89 \\
\hline Namulenga & 3,40 & 6,07 & 6,53 & 6,97 & 5,53 & 8,03 \\
\hline Puebla Criolla & 3,77 & 6,60 & 5,07 & 9,03 & 4,39 & 7,41 \\
\hline Nyiramuhondo & 4,87 & 7,07 & 6,68 & 8,79 & 5,68 & 5,97 \\
\hline CODMLV 059 & & 4,87 & & 19,56 & & 5,87 \\
\hline Nain de Kyondo & 3,67 & & 16,7 & & 5,26 & \\
\hline
\end{tabular}

Le nombre de graines par gousse a été plus élevé en saison B qu'en saison A sur tous les sites, pour les mêmes raisons invoquées pour le nombre de gousses que les autres variétés. Ce paramètre est très important car il influence le rendement. Bien qu'étant un caractère variétal, il peut être influencé par les conditions du milieu, surtout au moment du remplissage des gousses.Si le nombre de graines par gousse n'a pas varié en saison A à Mulamba, en saison B par contre les variétés Nyiramuhondo et Puebla Criolla ont enregistré plus de graines par gousse. A Kavumu, l'interaction variete-saison a classé les variétés M 211, Puebla Criolla et nyirasmuhondo en tête en saison $\mathrm{B}$ pour le nombre de graines par gousse. La même constatation a été faite pour Kalehe où les variétés Nyiramuhondo, M 211, Cuarantino 0817 et Puebla Criolla ont enregistré plus de graines par gousse.

4.2.4 Rendement des variétés par saison et par site. Le rendement en graines des variétés en étude par saison et par site est présenté au tableau 10.

Tableau 10 : Rendement (en $\mathrm{t} / \mathrm{ha}$ ) des variétés par saison et par site

\begin{tabular}{|l|l|l|l|l|l|l|}
\hline \multirow{2}{*}{ Variétés } & \multicolumn{2}{l|}{ Mulamba } & \multicolumn{2}{l|}{ Kavumu } & \multicolumn{2}{l|}{ Kalehe } \\
\cline { 2 - 7 } & Saison A & Saison B & Saison A & Saison B & Saison A & Saison B \\
\hline M 211 & 0,173 & 1,346 & 0,198 & 3,850 & 0,770 & 2,020 \\
\hline Cuarantino 0817 & 0,220 & 0,878 & 0,262 & 3,200 & 0,900 & 1,590 \\
\hline Namulenga & 0,373 & 1,063 & 1,060 & 4,960 & 1,400 & 2,080 \\
\hline Puebla Criolla & 0,371 & 0,923 & 0,214 & 4,170 & 0,840 & 1,310 \\
\hline Nyiramuhondo & 0,304 & 2,437 & 0,350 & 4,910 & 1,190 & 1,230 \\
\hline CODMLV 059 & & 0,885 & & 4,300 & & 1,880 \\
\hline Nain de Kyondo & 0,360 & & 0,100 & & 0,980 & \\
\hline
\end{tabular}


Le rendement en graines des variétés en étude a été beaucoup plus élevé en saison $B$ qu'en saison A sur tous les sites, à cause des conditions de pluviométrie. Ainsi cette saison peut être considérée comme la plus favorable pour le haricot volubile. Ce rendement a dépassé à Kavumu le potentiel des variétés en station à cause des sols riches de cette contrée, sols basés sur des laves volcaniques. Dans les autres sites le rendement a été dans la marge du milieu réel et l'a même dépassé pour certaines variétés. Au cours des saisons A et B le rendement n'a pas varié entre les variétés à Mulamba. A Kavumu, l'interaction variétésaison a donné le rendement le plus élevé en saison B aux variétés Namulenga et Nriramuhondo, suivies en saison B par les variétés Puebla Criolla, M 211 et Cuarentino. A Kalehe, ce sont les variétés Puebla Criolla, Nyiramuhondo et M 211 en saison B qui ont donné plus de rendement que les autres variétés. Les variétés CODMLV 059 et Nain de Kyondo ont été comparées aux autres au cours de la saison où elles étaient en essai par le test $t$ de Student; les résultats ont été les suivants : à Kavumu, Nain de Kyondo a montré un rendement inférieur aux autres, alors qu'il a été égal aux autres mais inférieur à M 211 ; CODMLV 059 a été égal aux autres variétés à Kavumu et Kalehe.

\section{CONCLUSIONS ET RECOMMANDATIONS}

Les résultats de ce travail qui a comparé sept variétés de haricot volubile cultivées au cours de deux saisons successives (saison A 2017 et saison B 20180) à Mulamba, Kavumu et Ihusi ont montré que les paramètres végétatifs (hauteur des plants et nombre de feuilles) et ceux de rendement étaient plus élevés en saison $\mathrm{B}$ qu'en saison $\mathrm{A}$ et que dès lors, la saison $\mathrm{B}$ peut être considérée comme la plus favorable au haricot volubile dans ces sites. Sur le plan du rendement, il y a eu des variations selon les sites mais les variétés suivantes peuvent être recommandées : Nyiramuhondo, Namulenga et Puebla Criolla, pourvu que bien entendu elles puissent présenter de bonnes qualités culinaires et sensorielles.

\section{REFERENCES BIBLIOGRAPHIQUES}

Baudouin, J.; Vanderborght, T.; Kimani, P. \& Mw'angombe, A., 2001. Légumineuses à graines: Haricot: 337-355. In : Raemekers, R. (ed.) : Agriculture en Afrique tropicale, Bruxelles, DGCI : 1423 pp

Carburet, A. et Hekimian, C., 2003. Les légumineuses à graines : 865-878. In : Memento de l'agronome, Paris-France, CIRAD-GRET : 1698 pp

Civava, M., 2013. Amélioration des agrosystèmes intégrant le haricot commun (Phaseolus vulgaris L.) au Sud Kivu montagneux. Gestion durable des agrosystèmes en milieu tropical bumide. https://www.orbi.uliege.be/handle/226 8/164008: 23 pp le 17/4/2018

Du Jardin, 1994. Respiration et autres catabolismes oxydatifs: 430-450. In : Agronomie moderne: bases

physiologiques et agronomiques de la production végétale : $543 \mathrm{pp}$

INERA, 2006. Caractérisation du haricot commun (Phaseolus vulgaris L.). Programme national haricot $(\mathrm{PNH})$, Centre de Mulungu : 58 pp

Nieuwenhuis, R et Nieuwelink, J., 2005. La culture du soja et d'autres légumineuses. Digigrafi, Wageningen, Pays Bas: 75 pp

Robertson, R., 2017. Then 9 healthiest beans and legumes you can eat. Healthline. https://www.healthline.com/nutrition/ healthiest-beans-legumes: 8 pp le $9 / 7 / 2018$

Vogel, S., 2017. Checking the pulse prices under pressure. Rabo Research., https://www.research.rabobank,com/fa r/en/sectors/grains-oilseeds/checkingthe-pulse-global-pulse-prices-underpressure.html :3 pp le5/8/2018 
Harvestplus, 2009. Le haricot riche en fer. https://www.unsen.org:3 pp le $16 / 8 / 2018$

Tsibingu, M.; Mukadi, T.; Mpoyi, M. ; Ntatangolo, B. ; Musenge, D.; Tsibingu, L. ; Kazadi, N. ; Nyembo, N et Mushumbani, M., 2017Evaluation de la productivité du maïs (Zea mays L.) sous amendement organique et minéral dans la province du Lomami, République Démocratique du Congo. Journal of Applied Biosciences, 109 (1) :10571-10579.

Vwima, S., 2014. Le rôle du commerce frontalier des produits alimentaires avec le Rwanda dans l'approvisionnement des ménages de la ville de Bukavu (province du Sud Kivu). Thèse universitaire Wallonie-Europe, Université de Liège-Gembloux : 195 pp Walangululu, M.J.; Balume, K.I. ; Mulu, L. \& Nziguheba, G., 2007. Final report of legume choice project. GIZ-BMZ project. Bukavu, DRC: 22 pp 